\title{
From Our Practices to Yours: Key Messages for the Journey to Integrated Behavioral Health
}

\author{
Stephanie B. Gold, MD, Larry A. Green, MD, and CJ Peek, PhD
}

Background: The historic, cultural separation of primary care and behavioral health has caused the spread of integrated care to lag behind other practice transformation efforts. The Advancing Care Together study was a 3-year evaluation of how practices implemented integrated care in their local contexts; at its culmination, practice leaders ("innovators") identified lessons learned to pass on to others.

Methods: Individual feedback from innovators, key messages created by workgroups of innovators and the study team, and a synthesis of key messages from a facilitated discussion were analyzed for themes via immersion/crystallization.

Results: Five key themes were captured: (1) frame integrated care as a necessary paradigm shift to patient-centered, whole-person health care; (2) initialize: define relationships and protocols up-front, understanding they will evolve; (3) build inclusive, empowered teams to provide the foundation for integration; (4) develop a change management strategy of continuous evaluation and course-correction; and (5) use targeted data collection pertinent to integrated care to drive improvement and impart accountability.

Conclusion: Innovators integrating primary care and behavioral health discerned key messages from their practical experience that they felt were worth sharing with others. Their messages present insight into the challenges unique to integrating care beyond other practice transformation efforts. ( $\mathrm{J}$ Am Board Fam Med 2017;30:25-34.)

Keywords: Delivery of Health Care, Integrated, Mental Health, Practice-based Research, Primary Health Care

Of adults with a behavioral health $(\mathrm{BH})$ disorder, $67 \%$ do not receive adequate treatment. ${ }^{1} \mathrm{Un}$ treated $\mathrm{BH}$ conditions result in avoidable suffering and increase the cost of care through lack of medical improvement, decreased adherence to treatment, and inappropriate health care utilization. ${ }^{2}$ Most patients with $\mathrm{BH}$ conditions, including children, are seen in medical settings, most commonly primary care (PC), presenting the need and oppor-

This article was externally peer reviewed.

Submitted 17 March 2016; revised 1 September 2016; accepted 7 September 2016.

From the Department of Family Medicine, University of Colorado School of Medicine, Aurora, CO (SBG, LAG); Department of Family Medicine and Community Health, University of Minnesota School of Medicine, Minneapolis, $\mathrm{MN}$ (CJP).

Funding: The data for this paper comes from the Advancing Care Together (ACT) study which was sponsored by the Colorado Health Foundation (CHF-3848).

Conflict of interest: none declared.

Corresponding author: Stephanie B. Gold, MD, Department of Family Medicine, University of Colorado, 12631 East 17th Ave, Mail Stop F496, Aurora, CO 80045 (E-mail: stephanie.gold@ucdenver.edu). tunity to replace separated systems of care that do not adequately meet the needs of patients with integrated, "whole-person" care.

Integrated behavioral health (or integrated care) is the care that results from a practice team of PC and $\mathrm{BH}$ clinicians working together with patients and families, using a systematic and cost-effective approach to provide patient-centered care for a defined population. This care may address mental health, substance use conditions, health behaviors (including their contribution to chronic medical illnesses), life stressors and crises, stress-related physical symptoms, and ineffective patterns of health care utilization. ${ }^{3}$ The historic, cultural separation of training, payment, and delivery systems for PC and $\mathrm{BH}$ have caused the spread of integrated care to lag far behind other practice transformation efforts (eg, the Patient Centered Medical Home [PCMH]). New payment structures and delivery models that support the integration of $\mathrm{PC}$ and $\mathrm{BH}$ are emerging, facilitating the movement toward integrated care as part of the drive to achieve the 
Triple Aim of better patient experience, lower costs, and improved population health. ${ }^{4,5,6}$

The Advancing Care Together (ACT) study was a demonstration and evaluation project involving 11 diverse practices in Colorado pursuing their own ideas about how to integrate care under local conditions, using available resources. ${ }^{7,8}$ After practice leaders from these rural and urban, large and small, public and private practices worked together for 3 years, through conflicts, employee turnover, and crises, they convened for 2 days to reflect on the practical lessons they had learned about integrating $\mathrm{BH}$ and $\mathrm{PC}$ to meet the needs of patients. They accepted the responsibility to candidly debate and discern messages for other practices considering the pursuit of better care for their patients through integration.

Prior $7 A B F M$ publications from ACT described 5 organizing constructs that shape integrated behavioral health' : (1) the REACH, or the extent to which services are delivered to the target popula$\operatorname{tion}^{10}$; (2) the physical layout of the integrated work space ${ }^{11}$; (3) the approach to patient transitions via warm handoffs or referrals ${ }^{12}$; (4) the care pathways for varying severity of illness; and (5) a shared mental model. These were published in a supplement along with 4 other articles that contend that successful integration requires: (1) appropriate workforce preparation ${ }^{13} ;(2)$ integrated electronic health records (EHRs) ${ }^{14}$; (3) adequate staffing ratios and flexible scheduling ${ }^{15}$; and (4) external financing to cover the associated costs to the practice. ${ }^{16}$ Editorials and commentaries in the supplement relate these findings to policy, ${ }^{17}$ practice transformation research, ${ }^{18}$ and leadership theory. ${ }^{19}$

These prior publications represented the analysis of empirical observations by the ACT evaluation team, and, in the case of commentaries and editorials, the viewpoints of external experts. In contrast, this article examines a previously unanalyzed data set of direct messages from practice leaders: experts in how integration is cultivated on the ground. The purpose of this article is to report these messages from the ACT innovators to those who may follow in their footsteps.

\section{Methods}

\section{Practices}

The ACT study was sponsored by the Colorado Health Foundation and administered by the De- partment of Family Medicine at the University of Colorado-Denver with a steering committee of national and local experts and an independent evaluation conducted by a team at Oregon Health \& Science University. Clinicians and practice staff were invited to propose their own ideas for integrating $\mathrm{BH}$ and $\mathrm{PC}$, and the steering committee selected a diverse sample of 11 practices, including both community mental health and PC practices. Each practice received up to $\$ 150,000$ over 3 years to enable their participation in the study. ${ }^{8}$

\section{Data Collection}

At the end of 3 years of participation in the ACT study that included multiple collaborative meetings among the practices and local site visits, practice leaders (referred to as "innovators" throughout the remainder of this article) from the sites came together for a closing meeting on September 12-13, 2014. This closing meeting was designed to systematically harvest the most important lessons learned by the innovators in all 11 practices, distilled into practical messages that the innovators wanted to pass on to others pursuing integrated care. Data were collected in 4 stages: (1) practices reflected together on the 3-year experience; (2) 6 simultaneous small group conversations produced 6 sets of written messages; (3) the larger group synthesized the small group messages into clearer and more powerful messages embraced by all; and (4) the authors synthesized and organized these messages for transmission to others on behalf of those practices. The first 3 stages of this process were planned in advance, with stages 2 and 3 facilitated systematically.

Stage 1: Warm-up reflections in the full group. An innovator from each practice was asked in advance to comment on day 1 about the approach their practice pursued, how they would "sell" integration, how best to set up leadership, advice to others seeking to integrate care in their organization, communication strategies, and what policy changes they felt were most needed. These were captured in notes by group facilitators for innovators to review overnight before stage 2 .

Stage 2: Crafting messages in small groups. Over 4.5 hours the following day, the innovators articulated the most important messages for future implementers. Innovators were divided into 6 facilitated workgroups of 8 to 10 that included patient representatives and members of the ACT program 
office, steering committee, and evaluation team assisting as requested to help the innovators crystallize their lessons and messages. The first phase asked "What did you learn as an innovator that you most want to leave for others who may follow in your footsteps - lived experience for others to load their own experiments for success?" The second phase involved turning "what we learned" into specific practical messages. The messages were prioritized to consensus within each small group for report to the full group the next day. A recorder in each group captured the work on flipcharts, worksheets, or typed notes.

Stage 3: Large group synthesis. For 90 minutes on the final morning, the full group articulated common themes, along with significant differences, in the messages created by all 6 groups. In the first phase, each small group reported out their messages and answered any questions of clarification. The task for all was to simply listen - knowing that the next phase would be to identify what was in common across messages, connect them, and enrich them with the insights of the entire group. In this second phase, the facilitator led a large group discussion that synthesized the messages, adding nuance and detail as appropriate. This synthesis discussion was audio-recorded and transcribed.

\section{Analysis}

The analysis was informed by a general inductive approach: to condense the textual data into brief summary format and develop a message framework reflecting the underlying experience of the innovators. ${ }^{20}$ This was done via immersion/crystallization, ${ }^{21}$ as each of the authors separately immersed themselves in all of the source data, noting themes as they emerged. The authors then came together to resolve discrepancies in their crystallized themes in iterative cycles until consensus was reached, returning to the raw textual data regularly to ensure that fidelity to the original messages and language was preserved.

Thus, the analysis was in effect a 3 -stage process of crystallization: (1) innovators reflected on their experiences to crystallize key messages in small groups; (2) the facilitated large group summary report-out crystallized themes across groups and delved into the relationships of the themes with one another; and (3) the authors immersed themselves in all of the textual data to organize and crystallize the final set of messages.

\section{Results}

Five main themes captured the messages from ACT practices to those who might follow in their footsteps: (1) frame integrated care as a necessary paradigm shift to patient-centered, whole-person health care; (2) initialize: define relationships and protocols up front, understanding they will evolve; (3) build inclusive, empowered teams to provide the foundation for integration; (4) develop a change management strategy of continuous evaluation and course correction; and (5) use targeted data collection pertinent to integrated care to drive improvement and impart accountability. The idea of the paradigm shift is a global orientation to an innovative process that displaces practice notions that integrated care can be accomplished by a few small adjustments in customary practice. The initialization work precedes and prepares for change but also extends into the subsequent concepts (Figure 1). Each theme is presented below with a brief explanation and key messages from the innovators that defined them.

\section{Frame integrated care as a necessary paradigm shift to patient-centered, whole-person bealth care}

a) Eliminate the division between physical and mental health at the clinical and organizational level to better meet patient needs.

"We need to stop talking about mental and physical bealth; there is just health. It needs to be delivered and paid for as one."

"Whole-person health care starts and ends with the Triple Aim: better bealth, better care, lower costs."

"Enable flexible, personalized, cost-effective, seamless care."

"Adopt patients' needs as the organizing principle of service delivery."

"Integration allows us to treat our patients at multiple levels—so much more than being either a PC or BH provider."

"Integration is good for payers, providers, and patients. Period."

b) Treat integration as the conceptual and operational framework for the entire organization rather than a separate initiative.

"We're not talking about altering, amending, [or] changing. We're talking about transforming the way we do business." 
Figure 1. Lessons learned by early innovators on how to integrate care in your practice: relationships between main themes captured from participants in the Advancing Care Together study at their closing meeting, September 2014.

\section{Frame integrated care as a necessary paradigm shift to patient-centered, whole-person health care \\ a) Eliminate the division between physical and mental health at the clinical and organizational level to better meet patient needs \\ b) Treat integration as the conceptual and operational framework for the entire organization rather than a separate initiative}

Initialize - define relationships and protocols up-front, understanding they will evolve

a) Create a shared vision using common language that everyone understands

b) Create and verify consensus regarding what partnerships entail

c) Establish standard processes and infrastructure necessary for your integrated care approach: workflows, protocols for scheduling and staffing, documentation procedures, and an integrated EHR

d) Determine the practice's risk tolerance, pursue funding opportunities, and commit to your integration approach
Build inclusive, empowered teams as the foundation for integration

a) Create inclusive care teams, centered around the patient and their needs, where all members have an equal voice

b) Invest in relationship- and trust-building among team by scheduling regular multidisciplinary, interprofessional communication

c) Find the right people for the team with the necessary skillsets, experience, and mentality

d) Identify leaders at all levels

Develop a change management strategy of continuous evaluation and coursecorrection

a) Create a culture open to learning from failure

b) Cultivate support for change within and outside of the practice

c) Encourage a broader-scale call for integration by engaging patients early and often

Use targeted data collection pertinent to integrated care to drive improvement and impart accountability

a) Collect data on defined, priority outcomes to measure your progress toward integrated care and also to demonstrate the value of integrated care to external stakeholders

b) Create feedback loops for data to inform quality improvement efforts

c) Report data internally both at the level of the practice for shared accountability and at the individual provider level to motivate change 
"Integration is not a program or a person. It is a paradigm shift."

"Let integrated care show everywhere."

\section{Initialize: define relationships and protocols up front, understanding they will evolve}

Consider the use of a template or "starter kit" for a successful beginning, but balance this with the knowledge that the process of integration will vary by practice, change over time, and "there is no one right way to do this."

a) Create a shared vision using common language that everyone understands.

To establish consensus, "bring everyone to the table ... [and] listen to the naysayers." Assure not only shared language but also shared meanings.

"Know where you are at on the integration curve, where you want to get to, and what that means to you all....Take the time before the beginning to talk it through, who is going to need to do what and set in place a procedure to manage the changes."

"It's not just the language, the language is not enough in and of itself; It's what it means. Make sure that the understanding is not just the words and use of the words but that everybody is on board with what they mean by those terms."

"If you forget to write it down you can get mistaken assumptions."

b) Create and verify consensus regarding what partnerships entail.

Be clear on the terms of any partnerships at the start to avoid confusion and misunderstanding later, recognizing that the histories, cultures, and operating arrangements of $\mathrm{BH}$ and $\mathrm{PC}$ practices are different.

"Negotiate and define the comprehensive clinical and business relationship at the beginning and do this in the depth that your activities require. This is a start at building trust."

"Be clear about terms of the partnership at the macroorganizational and microclinical level."

"Talk about the 'what ifs'-time to reflect, like premarital counseling-what might this really be like and how will we solve these things along the way."

c) Establish standard processes and infrastructure necessary for your integrated care approach: workflows, protocols for scheduling and staffing, documentation procedures, and an integrated EHR.

Build workflows and protocols that take into account how data will be obtained and recorded.
Make scheduling flexible enough to accommodate the needs of patients and the demands placed on $\mathrm{PC}$ providers while respecting the time of the $\mathrm{BH}$ provider. Include EHR templates for $\mathrm{BH}$ in documentation procedures.

"What is really critical is building your structure so people can do the tasks you want them to do."

"Get an EHR that meets the needs of those in this negotiated relationship, and be prepared for the expense and time it takes to work."

d) Determine the practice's risk tolerance, pursue funding opportunities, and commit to your integration approach.

Create business plans that consider how much the practice would be willing to lose in terms of finances and human capital and then invest in the model; do not wait for payment reform to catch up to the clinical evidence.

"Do not let the funding stop you: stick to your vision."

"Go ahead and be the change you want to see now: develop workarounds for the obstacles."

\section{Build inclusive, empowered teams to provide the foundation for integration}

a) Create inclusive teams, centered around the patient and their needs, where all members have an equal voice.

Incorporate all roles of clinic staff into the team, from $\mathrm{BH}$ and PC providers to the Board of Directors, medical assistants, front desk, and maintenance staff. View the levels of the team as having equal standing and role to play, not as a "top-down" hierarchy. In particular, avoid hierarchy between $\mathrm{PC}$ and $\mathrm{BH}$ providers.

"The patient is the center of the team, so know your patients and their circumstances."

"The patient should be the driver."

"Make sure every provider has the chance to work in an integrated fashion as a team member."

"One reason we see these practices fail is because PC just hires a BH provider, and that is not a partnership... BH providers would be involved in planning and organization, $B H$ would have more care coordination involvement, $B H$ has a vote in the organization in structure and management of money... it is less of a top-down approach from PC to BH."

b) Invest in relationship- and trust- building among team members by scheduling regular multidisciplinary, interprofessional communication.

"If you don't trust your [team] members, it's not going to work." 
"Invest in building and training integrated care teams to create trust among patients, providers, staff, and management for integrated BH in PC. Trust empowers integrated care."

"Meet regularly with your team (inclusively defined), because it makes everything go better. It's our patient, not yours or mine."

"In places that thrust BH folks or vice versa thrust $P C$ people into a setting where there is really no attention to relationship building and understanding of roles etcetera, you are not likely to be successful."

c) Find the right people for the team with the necessary skill sets, experience, and mentality.

$\mathrm{BH}$ providers with integrated care training are not being produced by the current education system at the rate they are needed; this is something that needs to be addressed in the long term.

"The partnership is critical and finding the right partners is essential. It is okay to start again to find the right partner and not everybody is the right fit for the practice."

"Bring in people who have been trained in integrated care, BH providers who have worked in integrated care."

"You have to have staff that can deal with the new paradigm. That they were successful in the old paradigm does not necessarily predict that they will be successful in the new paradigm."

"An involved and empowered practice manager with a significant skill set is key to belp projects unfold well and for your projects to be accountable to the success of the practice."

d) Identify leaders at all levels.

Having leaders at every level supports building trust throughout the organization; when the vision for integration comes from the top of the organization but is not shared at every level it can falter, but people who are trusted from each level championing the message foster trust in that message.

"Have champions at every level and across $\mathrm{BH}$ and PC."

"The clinicians and the staff champions have somewhat different functions but it is really, really important they are out there or else they are going to fail."

\section{Develop a change management strategy of continuous evaluation and course correction}

While initial agreements, protocols, and relationship definitions are created to enable a successful start, lived experience will necessitate changes over time. Plan ahead to manage these changes with intentionality. a) Create a culture open to learning from failure.

"Do not be afraid of failure: our best lessons (solutions) come from our failures."

"Know that many iterations are likely necessary for change to occur and it is messy, and may involve crises that allow change to occur."

"Leaders need to allow the team to innovate: to fail, succeed, adjust, and evolve as needed and that it be a safe place for that to happen in a transparent way."

b) Cultivate support for change within and outside of the practice.

Share success stories and maintain focus on value systems such as relationships and personal meaning to maintain momentum. Develop a support system at all levels of the organization as well as in external partners to prevent and address barriers to progress.

"Reinforce adoption of integration through success stories, deal all staff into what you are doing and why: including patient stories."

"The support and stimulation that is needed such as from ACT is so important in this challenging context. Find yourself a great support system: people thinking about this and doing this. I have seen practices struggle without support from the top. Our Board is very supportive and I don't know how we could have made progress without that."

c) Encourage a broader-scale call for integration by engaging patients early and often. Innovators believe that patient demand for integrated care is lacking, and that a broader call for change is necessary. Involve patients from the start to help build awareness and support.

"We want patients to demand the care."

"If we have patients with us from the beginning it will both better inform what we do but also build that movement. It's a mistake to start without patients. It's an opportunity to start with patients."

\section{Use targeted data collection pertinent to integrated care to drive improvement and impart accountability}

a) Collect data on defined, priority outcomes to measure your progress toward integrated care and also to demonstrate the value of integrated care to external stakeholders.

"Practice level data must be accurate, usable, and timely."

"You need to demonstrate doing a good job because people are watching and expecting results and it is about 
all you do. Define outcomes broadly: population level outcomes, clinical outcomes, satisfaction, patient experience, cost: showing these is key to the sustainability of an integrated model."

b) Create feedback loops for data to inform quality improvement efforts.

Choose relevant measures, establish processes to obtain and analyze data, and determine your methods to quickly and routinely apply what your analysis shows to improve future performance.

"YYou] have to look at data, but also turn data and results into actual change."

c) Report data internally both at the level of the practice for shared accountability and at the individual provider level to motivate change.

"You succeed or fail together. It's not BH providers well you didn't do that or so that's why that failed, or PC providers you did not do that so that is why that failed. It's about team: so keeping it at the practice level and what are all the pieces that went into that success or that failure."

"Provider-specific data really works: no one wants to be on the bottom. If you think you don't have any $B H$ need on your panel, are all diabetic patients doing well? If not, why not? Until they saw that graph, saw their numbers on it, did not want to be on the bottom of it, they saw that $10 \%$ is medicine and the rest is environment, behavior, and what we got from our parents."

\section{Discussion}

These lessons learned by the ACT innovators in integrating $\mathrm{BH}$ and $\mathrm{PC}$ are focused insights into "on the ground" integration from a perspective that is not easily gleaned from practice checklists or the literature. The innovators created these key messages, expressed here in their original language, for use by other practice leaders. Existing integrated care checklists provide the "what" that practices must accomplish; the themes in this article contextualize the "what" with the "why" and "how." The themes mirror, in a complementary fashion, recently articulated pathways to in-

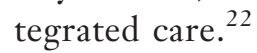

While some of these findings may be familiar to leaders and practices with experience in practice redesign, these messages also present insight into the unique challenges of integrating behavioral health care. For example, previous research on the transition to the PCMH has described the importance of relationships in practice transformation; healthy relationships provide the foundation for change to occur. ${ }^{23}$ However, while the PCMH model has gained acceptance and spread over the last decade, the movement toward integrated care has seen slow progress over 40 years. A sociological shift in relationships is required for integrated care: one that involves the culture of health care providers and even how medicine itself is viewed. Physical and behavioral health — and the providers of such care-are united on even ground in integration, and the extent to which this is a radical change should not be underestimated. The ACT innovators realized integrated care presents a special case in practice transformation; their messages indicate the need for dramatic shifts in relationships, mindset, and purpose.

These shifts to equalized relationships, a mindset of whole-person health, and a set purpose of truly meeting patients' needs are foundational changes, and changes of such magnitude make adaptive leadership essential. In adaptive leadership, solutions stem from the collective intelligence of all team members rather than upper-level management. ${ }^{24}$ Adaptive leaders support their team, rather than direct them. The innovators' messages on identifying leaders at all levels and ensuring all members of the team have an equal voice echo these principles.

Although other practice transformation efforts have been grounded in usual continuous quality improvement and technical adjustments to an existing paradigm, the new paradigm of integrated care requires that these foundational shifts occur first to enable quality improvement to be successful. However, continuous quality improvement is still an essential part of the transformation to integrated care, fitting under the theme, "use targeted data collection pertinent to integrated care to drive improvement and impart accountability." Many rapid-cycle methods for continuous quality improvement (eg, 6-sigma, lean, Plan-Do-Study-Act [PDSA]) have been articulated for use in healthcare settings ${ }^{25}$; the ACT innovators did not reference 1 methodology as preferred in particular but instead highlighted the need for developing a plan for continuous feedback loops from the beginning.

These 11 practices in Colorado comprised a purposeful sample of diverse settings, ranging from rural to urban, Federally Qualified Health Centers to private practices, and Mental Health Centers to PC clinics. The practices all took an adaptive rather than prescribed approach to integration; as such, these 
Table 1. Lessons Learned by Early Innovators on How to Integrate Care in Your Practice: Selected Practical Examples from Participants in the Advancing Care Together Study at Their Closing Meeting, September 2014

\begin{tabular}{lll}
\hline Message & Example \\
\hline
\end{tabular}

1. Frame integrated care as a necessary paradigm shift to patient-centered, whole-person health care

a. Eliminate the division between physical and mental health at the clinical and organizational level to better meet patient needs

b. Treat integration as the conceptual and operational framework for the entire organization rather than a separate initiative

2. Initialize-define relationships and protocols up-front, understanding that they will evolve

a. Create a shared vision using common language that everyone understands

b. Create and verify consensus regarding what partnerships entail

c. Establish standard processes and infrastructure necessary for your integrated care approach: workflows, protocols for scheduling and staffing, documentation procedures, and an integrated EHR

d. Determine the practice's risk tolerance, pursue funding opportunities, and commit to your integration approach

3. Build inclusive, empowered teams as the foundation for integration

a. Create inclusive care teams, centered around the patient and their needs, where all members have an equal voice

b. Invest in relationship- and trust building among team members by scheduling regular multidisciplinary, interprofessional communication

c. Find the right people for the team with the necessary skill sets, experience, and mentality

d. Identify leaders at all levels

4. Develop a change management strategy of continuous evaluation and course-correction

a. Create a culture open to learning from failure

b. Cultivate support for change within and outside of the practice

c. Encourage a broader-scale call for integration by engaging patients early and often

5. Use targeted data collection pertinent to integrated care to drive improvement and impart accountability

a. Collect data on defined, priority outcomes to measure your progress toward integrated care and also to demonstrate the value of integrated care to external stakeholders

b. Create feedback loops for data to inform quality improvement efforts

c. Report data internally both at the level of the practice for shared accountability and at the individual provider level to motivate change

EHR, electronic health record.

Ask providers to discuss both failures and successes at meetings with their peers to normalize failure within the process of change.

Develop a plan for increasing awareness amongst patients about integration and available services (possible themes for patient messaging could include "one-stop shop," "we specialize in all of you," "whole-person care.")

Routinely provide outcomes data to individual providers on their performance compared to practice averages as well as target levels.

Put the shared vision and agreements in writing to help prevent future ambiguity. that can generate revenues to sustainably continue the model, such as billing for case management.

Include $\mathrm{BH}$ providers in planning meetings regarding organization and finances.

te gular (at minimum biweekly) meetings for planning, and/or morning huddles.

Hire a practice manager with not only office management skills but also relevant project management skills such as grant writing, negotiating insurance companies, and awareness of policy levers and barriers. 
real-world lessons likely have relatively strong external validity for other frontline practices. Another strength of these findings lies in the continuity of shared experience during a 3 -year period, leading to development of a high level of trust, candor, opportunities for self and group correction, and a sense of mission and duty to share hard-earned knowledge. Practice leaders, facilitators, and learning collaboratives striving to integrate care might use these key messages as a guide, perhaps allowing practices to avoid pitfalls experienced by early innovators and accelerate their transformation. (Table 1).

The authors gratefully acknowledge the hard work and dedication of the 11 practices participating in the ACT study: Axis Health System; Bender Medical Group, Inc.; Denver Health and Hospital; Jefferson Center for Mental Health; Kaiser Permanente Colorado; Midvalley Family Practice; Plan de Salud del Valle, Inc.; Primary Care Partners; Southeast Mental Health Services; University of Colorado Aging Center; and Westminster Medical Clinic.

To see this article online, please go to: http://jabfm.org/content/ 30/1/25.full.

\section{References}

1. Kessler RC, Demler O, Frank RG, et al. Prevalence and treatment of mental disorders, 1990 to 2003. N Engl J Med 2005;352:2515-23.

2. Kessler R, Stafford D. Primary care is the de facto mental health system. In: Kessler R, Stafford D, eds. Collaborative medicine case studies: Evidence in practice. New York: Springer, 2008; pp. 9-21.

3. Peek CJ, National Integration Academy Council. Lexicon for behavioral health and primary care integration: Concepts and definitions developed by expert consensus. Rockville, MD: Agency for Healthcare Research and Quality. AHRQ Publication No.13-IP001EF. April, 2013.

4. Katon WJ, Unützer J. Health reform and the Affordable Care Act: The importance of mental health treatment to achieving the Triple Aim. J Psychosom Res 2013;74:533-7.

5. Berwick DM, Nolan TW, Whittington J. The Triple Aim: Care, health, and cost. Health Aff 2008;27: 759-69.

6. Klein S, Hostetter M. In focus: Integrating behavioral health and primary care. Quality Matters. The Commonwealth Fund. 2014 Aug/Sept. Available from: http://www.commonwealthfund.org/publications/ newsletters/quality-matters/2014/august-september/ in-focus\#/. Accessed Dec 23, 2015.

7. Davis M, Balasubramanian BA, Waller E, Miller BF, Green LA, Cohen DJ. Integrating behavioral and physical health care in the real world: Early lessons from Advancing Care Together. J Am Board Fam Med 2013;26:588-602.
8. Green LA, Cifuentes M. Advancing Care Together by integrating primary care and behavioral health. J Am Board Fam Med 2015;28(Suppl 1): S1-S6.

9. Cohen DJ, Balasubramanian BA, Davis $M$, et al. Understanding care integration from the ground up: Five organizing constructs that shape integrated practices. J Am Board Fam Med 2015;28(Suppl 1): S7-S20.

10. Balasubramanian BA, Fernald D, Dickinson LM, et al. REACH of interventions integrating primary care and behavioral health. J Am Board Fam Med 2015; 28(Suppl 1):S73-S85.

11. Gunn R, Davis MM, Hall J, et al. Designing clinical space for the delivery of integrated behavioral health and primary care. J Am Board Fam Med 2015; 28(Suppl 1):S52-S62.

12. Cohen DJ, Davis M, Balasubramanian BA, et al. Integrating behavioral health and primary care: Consulting, coordinating and collaborating among professionals. J Am Board Fam Med 2015;28(Suppl 1):S21-S31.

13. Hall J, Cohen DJ, Davis M, et al. Preparing the workforce for behavioral health and primary care integration. J Am Board Fam Med 2015;28(Suppl 1):S41-S51.

14. Cifuentes M, Davis M, Fernald D, Gunn R, Dickinson P, Cohen DJ. Electronic health record challenges, workarounds, and solutions observed in practices integrating behavioral health and primary care. J Am Board Fam Med 2015;28(Suppl 1):S63-S72.

15. Davis MM, Balasubramanian BA, Cifuentes M, et al. Clinician staffing, scheduling, and engagement strategies among primary care practices delivering integrated care. J Am Board Fam Med 2015;28(Suppl 1):S32-S40.

16. Wallace NT, Cohen DJ, Gunn R, et al. Start-up and ongoing practice expenses of behavioral health and primary care integration interventions in the Advancing Care Together (ACT) program. J Am Board Fam Med 2015;28(Suppl 1):S86S97.

17. Miller BF. When frontline practice innovations are ahead of the health policy community: The example of behavioral health and primary care integration. J Am Board Fam Med 2015;28(Suppl 1):S98-S101.

18. Dickinson WP. Strategies to support the integration of behavioral health and primary care: What have we learned thus far? J Am Board Fam Med 2015; 28(Suppl 1):S102-S106.

19. deGruy FV. Integrated care: Tools, maps, and leadership. J Am Board Fam Med 2015;28(Suppl 1): S107-S110.

20. Thomas DR. A general inductive approach for analyzing qualitative evaluation data. Am J Eval 2006; 27:237-46. 
21. Crabtree BF, Miller WL, eds. Doing qualitative research. 2nd ed. Thousand Oaks, CA: Sage Publications, 1999; pp. 127-43.

22. Ratzliff A, Phillips KE, Sugarman JR, Unutzer J, agner EH. Practical approaches for achieving integrated behavioral health care in primary care settings. Am J Med Qual. 2015 [Epub ahead of print].

23. Crabtree BF, Nutting PA, Miller WL, et al. Primary care practice transformation is hard work:
Insights from a 15-year developmental program of research. Med Care 2011;49(Suppl):S28S35.

24. Heifetz RA, Laurie DL. The work of leadership. In: On leadership. Boston, MA: Harvard Business Publishing. 2001; pp. 35-48.

25. Varkey P, Reller MK, Resar RK. Basics of quality improvement in health care. Mayo Clin Proc 2007; 82:735-9. 